\title{
Dog Ownership, Perceived Social Supports and Stress Among University Students
}

\author{
Vei Kit Lee*, Ming Sing Chai \\ Faculty of Social Science, Arts and Humanities, Tunku Abdul Rahman University College, Kuala Lumpur, Malaysia
}

Email adress:

lee_vk@outlook.com (V. K. Lee), chaims@acd.tarc.edu.my (M. S. Chai)

To cite this article:

Vei Kit Lee, Ming Sing Chai. Dog Ownership, Perceived Social Supports and Stress Among University Students. American Journal of Applied Psychology. Special Issue: Psychology of University Students. Vol. 4, No. 3-1, 2015, pp. 45-50. doi: 10.11648/j.ajap.s.2015040301.18

\begin{abstract}
This study was conducted to determine whether there is a significant mean difference of perceived stress between dog owners and non-dog owners and whether there are significant correlations between different sources of perceived social supports and perceived stress. Participants were 116 undergraduate students in a Malaysian private university college. Among 116 participants, 44 were dog owners. Participants completed 10-item Perceived Stress Scale (PSS-10) and Multidimensional Scale of Perceived Social Support (MSPSS). The results indicated that there was a significant mean difference of perceived stress between dog owners $(N=44, M=19.57)$ and non-dog owners $(N=72, M=21.67)$. Among non-dog owners, a significant negative correlation was found between perceived social support from friends and perceived stress. However, among non-dog owners, no significant correlations were found between perceived stress and perceived social support from family, friends, significant others and dogs. Further research using a bigger sample size is needed to verify the relationship between perceived stress and perceived social support among dog owners.
\end{abstract}

Keywords: Dog Ownership, Perceived Social Supports, Perceived Stress, University Students

\section{Introduction}

There are 56.7 million of households in the United States owning a total of 83.3 million dogs [1]. In Asia-Pacific, dog ownership is growing fast at a rate of $13 \%$ since the year 2006 until 2011. In 2011, the statistics showed that there are 62 million of households in Asia-Pacific owning a dog [2]. In Malaysia, an increasing number of households are adopting dogs as pets [3]. Study has found that pets might be able to provide social support that is more effective than other humans because pets will never give negative evaluation but humans sometimes do [4]. One of the common reasons given by people for owning dogs is to reduce stress [5].

Stress is developed when one feels overwhelmed and perceives self as unable to cope with demands of the situation [6]. There are two types of stress, which are objective stress and subjective stress. An individual is facing objective stress when a disruptive event happens in one's life [7]. On the other hand, subjective stress or perceived stress is being referred to one's perception on meaning of life events [8]. Both objective and perceived stress can induce either positive or negative effects depending on the degree of stress being experienced. Optimal level of stress helps to facilitate one to perform well and focus on task [9]. In contrast, excessive stress can negatively affect one's mental and physical health [10]. An individual is expected to be a victim of stress if he or she is having one of these stress symptoms such as feeling depressed, difficulty in concentration, high blood pressure, easily fatigue, frequent neck or shoulder pain and anxiety among others [6].

Previous studies had shown that most undergraduate students were experiencing stress symptoms in their daily lives regardless of their gender and ethnicity $[6,11]$. Study has indicated that university students in Malaysia experienced moderate level of perceived stress [12]. Some undergraduate medical students in Malaysia were found to have high level of stress that warrants attention $[13,14]$.

The high level of stress among undergraduate students was reported to be triggered by various academic stressors and non-academic stressors [14]. The academic stressors include too many assignments, poor performance in examination, large amount of contents to be learned, difficulty in learning, and short of time for revision $[6,15]$. On the other hand, non-academic stressors are such as inadequate resources to access, financial difficulties, lack of sleep, overcrowded lecture halls, uncertainty about future, relationship with 
others and high expectation from family, friends or self [6, $11,16]$.

In view of the tremendous stress faced by university students and the increasing popularity of dog ownership among Malaysians, it is important to explore how dog ownership and social support are related to stress among university students. Thus, the present study is aimed to determine whether there is a significant mean difference of perceived stress between dog owners and non-dog owners and whether there are significant correlations between different sources of perceived social supports and perceived stress.

\section{Literature Review}

\subsection{Dog Ownership and Perceived Stress}

Most university students self-reported that they owned pet dogs and cats with the purpose to alleviate loneliness and stress because they were experiencing transitions period and being away from their family members [4]. Previous study showed that pet dogs were able to buffer against perceived stress of dog owners especially when their dogs fulfilled their social needs [17]. In assessing 33 stress-related symptoms that might be experienced in the past two weeks, pet owners were found to have less physical illnesses and stress-related symptoms as compared to non-pet owners [17].

In another study on cardiovascular reactivity and the presence of pets [18], pet owners were reported to have the lowest cardiovascular measure with the presence of their pets when they were performing active coping tasks (arithmetic tasks) and passive coping tasks (immersed hand in cold water). In contrast, non-pet owners were found to have the lowest cardiovascular measure when they were alone. However, both pet owners and non-pet owners were having the highest cardiovascular reactivity when spouses were being around [18].

Other study found that pet dogs could act as buffers against perceived stress of dog owners through increasing frequency of exercises among dog owners[19]. Brown and Rhodes [19] reported that dog owners aged from 20 to 80 years in Western Canada spent averagely 132 minutes more than non-dog owners on walking within a week. Similarly, findings by Staats, Sears and Pierfelice [20] concluded that male faculty members in a Midwestern university owned pets because pets motivated them to exercise. A study in Japan also indicated that walking with dogs was better in reducing stress than walking without dogs [21]. In addition, elders in Southern California who did not own pets were more likely to visit the doctors than the participants who owned pets as stressors in their life increased [22].

Similarly, the difference in the level of perceived stress at work place was reported between dog owners and non-dog owners [23]. Dog owners were reported to have lower level of perceived stress than non-dog owners when their dogs were present in the workplace. In contrast, dog owners also were likely to have higher level of perceived stress than non-dog owners when their dogs were absent in their workplace because the dog owners were worried about their dogs [23]. Furthermore, pet owners who brought their pets to workplace tend to perceive more benefits than non-pet owners or pet owners who leave their pets at home [24].

Overall, most of the studies supported that pet dogs could be served as buffers against perceived stress [17, 25, 20-21, 23] and cardiovascular response to stress [25, 18, 26]. However, in the absence of dogs, some dog owners showed higher level of perceived stress [23 - 26]. Therefore, the current study was conducted to answer this research question: Is there a significant mean difference of perceived stress between dog owners and non-dog owners?

\subsection{Perceived Social Support and Perceived Stress}

Stress was a result of an individual's appraisal on an occurred stressor and own resources or abilities to cope with the stressor [27]. In transactional theory of stress and coping, there were three types of appraisal, which are primary, secondary and reappraisal. Primary appraisal referred to an individual's judgment about meaning that one assigned to a situation as threat, harm or challenge. The secondary appraisal referred to the individual's evaluation of available resources and abilities to cope with demand of the situation. Lastly, reappraisal referred to continuing evaluation of primary and secondary appraisals as the situation happened [28].

Different individuals could also experience varying level of stress from a specific stressor due to individual's appraisal and perceived social supports that are available. Perceived social support refers to individuals' perceptions on availability of support from family, friends or significant others during occurrence of stressful events [29]. Other than availability of social support provided, degree of perceived social support also depends on degree of supportiveness that is being provided by a provider [30]. When one is being exposed to stressor, one's cortisol response can be lowered by social support received from friends, family and significant others [31]. Besides, the individual tends to have low anxiety levels after exposure to stressor when the individual is having secure attachment and social support [31]. Research has found that students tend to talk with their friends in order to relieve stress as they perceive the most social support from their friends [16]. Other than social support from other humans, pets such as dogs also can be one of the sources that provide social support [17].

Individuals could cope with stressful events with social supports that were being provided by family, friends, significant others or even pet dogs. For instance, a study with adolescents who were studying in Senior High Schools in Ghana indicated that perceived social support from family and friends protect individuals from academic stress by providing ideas to solve problems, promoting healthy behaviours or reduce over-reactions to perceived stress [32]. Study with university students in Jordan also showed that both perceived social support from family and friends had weak negative correlation with perceived stress but only 
correlation between perceived social support from friends and perceived stress was significant [33]. In addition, a study in Germany indicated that social support from best friends was able to act as buffer against stress by suppressing cortisol levels [34]. Similarly, study done by Chao [35] found that college students were willing to interact with family, friends and significant others in order to get assistance in the form of informational or emotional support during stressful situations College students with low level of perceived social support were likely to have higher level of perceived stress [35].

In transactional theory of stress and coping, social support that met the demands of the stressful situation will be effective in buffering stress because perception of the social support helped to reduce the individual's perceived stress [36]. Besides, individual's perceived social support was largely based on one's past experience of receiving effective enacted support that included others' words and behaviours that were related to the stressful situation [36]. Researchers in reference [37] conducted a study by using 14-item Perceived Stress Scale (PSS-14) and Multidimensional Scale of Perceived Social Support (MSPSS) with additional 4 items to measure perceived social support from dogs. Their results indicated that there were significant negative correlations between general social supports (family, friends and significant others) with perceived stress level but no significant correlation between perceived support from dogs and perceived stress level [37]. The researchers explained that perceived social support from dogs did not help to reduce stress that arises from problems because dogs only provided emotional support instead of solutions to solve problems.

Finding regarding the correlation between perceived social support from dogs and perceived stress was not consistently non-significant. For instance, a study with Miami University students found that dogs were able to reduce perceived stress because dogs were able to provide similar social support as human friends by alleviating negative feeling of being rejected [17]. As compared to men, women were more likely to own pets in order to gain social support from the pet and get through hard time [20]. One of the reasons for women to gain social support from dogs or cats was that they felt lonely without owning dogs or cats [20]. Findings from study in reference $[4,38]$ indicated that social support from dogs, not friends, reduced more cardiovascular responses during stressors because the dog owners were more worried about being evaluated by friends and the dog owners perceived their dogs as a source for providing non-evaluative social support.

In general, there were inconsistencies between past studies on the relationships between perceived social support (family, friends, and pet dogs) and perceived stress. Besides, there were only two studies on the relationship between perceived social support from significant others and perceived stress $[35,37]$. Therefore, the current study seeks to address this research question: Are there significant relationships between perceived social supports from different sources and perceived stress?

\section{Methodology}

\subsection{Participants and Procedures}

Participants were 116 undergraduate students (41 males and 75 females) from a local private university college located in Kuala Lumpur. The participants' age ranged from 18 to 28 years old $(M=20.10, S D=1.71)$. Among the participants, 44 were dog owners and 67 were non-dog owners. These participants were selected through convenience sampling to ease the administration of questionnaires and to increase the willingness of participants to complete questionnaires. The purpose of study was explained before questionnaires were administered to participants. Participants gave their written informed consent for their willingness to participate in the study without any rewards. Participants completed demographic information, 10-item Perceived Stress Scale (PSS-10) and Multidimensional Scale of Perceived Social Support (MSPSS). Data were collected and analysed using SPSS.

\subsection{Measures}

Perceived Stress Scale (PSS-10) consisted of 10 items measuring an individual's level of perceived stress in the last month [39]. Participants rated each item using 5-point Likert scale from 0 (never) to 4 (very often). PSS-10 consisted of four positive and six negative items. One of the items was "In the last month, how often have you felt that things were going your way?" For the four positive items, scores were obtained by reversing responses (for instances, $4=0,3=1,2$ $=2,1=3$ and $0=4$ ). The sum of all 10 items became the total score for perceived stress [11]. The Cronbach alpha coefficient for PSS-10 was reported at .742 in a study using Malaysian university students as participants [40]. Cronbach alpha coefficient for this study was .799 which was moderately high.

Multidimensional Scale of Perceived Social Support (MSPSS) was used to indicate the total amount of social support received from family, friends and significant others. MSPSS consisted of 12 items that included three sub-scales: perceived social support from family, friends, and significant others [29]. An example of the items is "I can talk about my problems with my family." 7-point Likert scale that ranges from 1 (very strongly disagree) to 7 (very strongly agree) was used to indicate the participants' perceived social support. The Cronbach alpha coefficient for MSPSS was .88, and for the three sub-scales were .87 for family, .85 for friends, and .91 for significant others [29]. An additional subscale that consisted of 4 items were created by Duvall Antonacopoulos and Pychyl [37] to measure perceived social support from pet dogs. Alpha coefficient for MSPSS with the additional subscale was .87 while the alpha coefficient for the four subscales that included family, friends, significant others and dog were $.81, .84, .77$ and .83 respectively [37]. In the current study, MSPSS had alpha coefficient of .899 while the four subscales (family, friends, significant others and dog) had alpha coefficient of .824, .796, .856 and .961 
respectively.

\section{Result}

\subsection{Dog Ownership and Perceived Stress}

The independent-samples t-test was used to analyse whether the level of perceived stress between dog owners and non-dog owners was different. Table 1 showed that there was a significant mean difference of perceived stress between dog owners and non-dog owners, $t(116)=-2.05, p<.05$. Dog owners had lower level of perceived stress $(N=44, M=19.57)$ than that of non-dog owners $(N=72, M=21.67)$.
Pearson correlation coefficient was used to analyse the correlation between perceived social support and perceived stress for both dog owners and non-dog owners. For dog owners (refer to Table 2), no significant correlations were found between perceived stress and perceived social support from family, friends, significant others or dogs. For non-dog owners (refer to Table 3), a statistically significant negative correlation was found between perceived social support from friends and perceived stress, $r(72)=-.42, p<.05$. No significant correlations were found between perceived stress and perceived social support from family, $r(72)=-.23, p>.05$, and significant others, $r(72)=-.16, p>.05$.

\subsection{Perceived Social Support and Perceived Stress}

Table 1. Independent-sample t-test for perceived stress and dog ownership.

\begin{tabular}{|c|c|c|c|c|c|c|c|}
\hline & Dog Ownership & $\mathbf{N}$ & Mean & Std. Deviation & t-test & ty of & \\
\hline \multirow{2}{*}{ Perceived Stress } & Dog Owner & 44 & 19.57 & 5.51 & $\mathrm{t}$ & $\mathrm{df}$ & Sig. (2-tailed) \\
\hline & Non-dog Owner & 72 & 21.67 & 5.27 & -2.05 & 114 & .043 \\
\hline
\end{tabular}

Table 2. Correlations between perceived social support and perceived stress for dog owners.

\begin{tabular}{lllll}
\hline & Perceived social support & Family & Friends & Significant others \\
\hline & Pearson Correlation & Dogs & -.06 & -.10 \\
Perceived Stress & Sig. (2-tailed) & -.25 & -.13 & .682 \\
& N & .103 & .389 & 44 \\
& M & 44 & 44 & 18.93 \\
& SD & 18.95 & 17.14 & 44 \\
\hline
\end{tabular}

$\mathrm{M}=$ Mean; $\mathrm{SD}=$ Standard Deviation; $\mathrm{N}=$ Sample size.

\section{Discussion and Conclusion}

The present finding shows that the level of perceived stress among dog owners was significantly lower than that of non-dog owners. The finding supports the view that owning a dog can help university students to alleviate their loneliness and stress as they went through their turbulent transitions period and stayed far away from their families [5]. By having a dog as a companion, one can feel less lonely and have his or her social need being fulfilled by the pet. As indicated by the study done by McConell, Brown, Shoda, Stayton and Martin [17], dog owners were less likely to experience stress related-symptoms than non-dog owners and their dogs were able to act as buffers against perceived stress especially when their social needs such as belongingness and meaningful existence were fulfilled by their dogs.

Table 3. Correlations between perceived social support and perceived stress for non-dog owners.

\begin{tabular}{lllll}
\hline & Perceived Social support & Family & Friends & Significant others \\
\hline & Pearson Correlation & -.23 & -.42 & -.16 \\
Perceived Stress & Sig. (2-tailed) & .056 & .000 & .193 \\
& N & 72 & 72 & 72 \\
& M & 18.67 & 17.32 & 18.93 \\
& SD & 5.09 & 4.87 & 5.29 \\
\hline
\end{tabular}

$\mathrm{M}=$ Mean; $\mathrm{SD}=$ Standard Deviation; $\mathrm{N}=$ Sample size.

Pet dogs, unlike other humans, were able to provide non-evaluative supports which can boost positive feelings. It was reported by Allen, Blascovich, and Mendes [18] that pet dogs and cats were able to elicit calmer response in pet owners by improving the owners' stress coping abilities by encouraging positive feeling states. Thus, participants who were university students possibly experience additional non-evaluative supports and companionship from their dogs as compared to non-dog owners, who received support from other humans. These non-evaluative emotional supports received from dogs can serve as protection against the adverse effects of academic and non-academic stressors experienced by students in their daily lives.

Another possible reason for dog owners to have lower level of perceived stress was because dog owners tend to exercise more than non-dog owners. Previous studies have shown that dog owners had a greater tendency to walk more than non-dog owners because their dogs motivated them to walk $[19,20]$. In addition, walking with dogs was better than walking without dogs because the presence of dogs may require interactions between dogs and dog owners which could induce surge in parasympathetic neural activity that help to stabilise and 
buffer against stress [21]. Therefore, students who own dogs may spend time after their classes or free time to go for a walk or play with their dogs. Their meaningful interaction with their dogs could have uplifted their emotional state and thus reduced their level of perceived stress.

For the relationship between perceived stress and perceived social support, the finding indicated that those non-dog owners who received more social support from friends had lower level of perceived stress. This finding was consistent with several previous studies [32 - 35]. When individuals are experiencing stress, they need supports from others who are willing to listen and talk to them [35]. The social supports given by family and friends in the form of providing solutions, reducing perception on importance of stressors and facilitating positive behaviors can be beneficial to health [32]. With the help of these social supports from friends, individuals may be able to perceive the stressor events as more controllable and less threatening, thus reducing the level of perceived stress [34].

No significant relationships were found between perceived social support from family and significant others with perceived stress among non-dog owners. Similarly, perceived social support from family, friends, significant others and dogs were not related to perceived stress among dog owners. These findings indicate that those university students, regardless of whether they have dogs, do not depend on support from family and significant others on handling issues related to their stress experiences. For those who have dogs, even perceived support from dogs and friends do not seem to influence their level of perceived stress. These non-significant results could be due to the small sample size for dog owners and therefore not able to give an accurate estimation of the relationships that exist between perceived social support and stress. A bigger sample size is recommended for future study to verify the relationship between the perceived social support and stress.

Although the present finding showed the significant difference in the level of perceived stress between dog owners and non-dog owners, the limitation of the study makes it impossible to prove that dog ownership has a buffer effect on perceived stress. The buffer effect of dog ownership on perceived stress could be moderated by the presence of dogs and other factors. As recommendation, other variables such as attitude of dog owners towards pets, types and degrees of support from various sources should be included in future study. The use of a longitudinal study and the inclusion of other variables will allow further study and provide clearer explanation on relationships between dog ownership, perceived social support, and perceived stress. Besides, researcher in current study was using convenient sampling by recruiting participants from the university college. As a result, most of the participants were Chinese and non-Muslims. Thus, the current findings will not be able to generalize to Muslim community as the community is less likely to own dogs due to their religion. Instead, the Muslim community is more likely to own cats as compared to dogs. As indicated in reference [22], there were differences between owning dogs and other pets such as cats. Therefore, other pets such as cats could be included in future studies.

In a nutshell, current finding has indicated that owning dogs is associated with lower level of perceived stress among university college students. This finding provides insight for other researchers, parents, educators and students on ways that can help to reduce perceived stress. Individuals may refuse to own a dog due to responsibility to take care of the dog. Thus, current finding could be used to encourage individuals to own a $\operatorname{dog}$ in order to have lower level of perceived stress. Furthermore, social support from friends can be served as protective factors for university college students from experiencing high level of perceived stress.

\section{References}

[1] American Pet Products Association. (2014). 2013-2014 APPA national pet owners survey statistics: Pet ownership \& annual expenses. Retrieved from http://www.americanpetproducts.org/press_industrytrends.asp

[2] Anuwong, W. (2012). Pet foods: Growth opportunities amidst crises and beyond. Retrieved from http://www.petfoodindustry.com/uploadedFiles/Euromonitorfi nalPFFAsia2012.pdf

[3] Euromonitor International. (2014). Dog food in Malaysia. Retrieved http://www.euromonitor.com/dog-food-in-malaysia/report

[4] Allen, K. M., Blascovich, J., Tomaka, J., \& Kelsey, R. M. (1991). Presence of human friends and pet dogs as moderators of autonomic responses to stress in women. Journal of Personality and Social Psychology, 61(4), 582-589.

[5] Staats, S., Wallace, H., \& Anderson, T. (2008). Reasons for companion animal guardianship (pet ownership) from two populations. Society and Animals, 16, 279-291.

[6] Agolla, J. E., \& Ongori, H. (2009). An assessment of academic stress among undergraduate students: The case of university of Botswana. Educational Research and Review, 4(2), 63-70.

[7] Wainstock, T., Anteby, E., Glasser, S., Shoham-Vardi, I., \& Lerner-Geva, L. (2013). The association between prenatal maternal objective stress, perceived stress, preterm birth and low birthweight. The Journal of Maternal-Fetal \& Neonatal medicine, 26(10), 973-977.

[8] Cohen, S., Tyrrell, D. A. J., \& Smith, P. A. (1993). Negative life events, perceived stress, negative affect, and susceptibility to the common cold. Journal of Personality and Social Psychology, 64(1), 131-140.

[9] LeBlanc, V. R. (2009). The effects of acute stress on performance: Implications for health professions education. Academic Medicine, 84(10), 25-33.

[10] WHO. (2006). Preventing suicide. Retrieved November 20, 2012 from http://whqlibdoc.who.int/publications/2006/9241594381_eng. pdf

[11] Lim, Y. M., Tam, C. L., \& Lee, T. H. (2013). Perceived stress, coping strategy and general health: A study on accounting students in Malaysia. International Refereed Research Journal, $4(1), 88-95$. 
[12] Rafidah, K., Azizah, A., Norzaidi, M. D. Chong, S. C., Salwani, M. I., \& Noraini, I. (2009). Stress and academic performance: Empirical evidence from university students. Academy of Educational Leadership Journal, 13(1), 37-51.

[13] Abdus, S., Rabeya, Y., Sheikh, M. A. B., \& Mainul, H. (2013). Stress among medical students in Malaysia: A systematic review of literatures. International Medical Journal, 20(6), 649-655.

[14] Al-Dubai, S. A. R., Al-Naggar, R. A., Alshagga, M. A., \& Rampal, K. G.. (2011). Stress and coping strategies of students in a medical faculty in Malaysia. Malaysian J Med Sci, 18(3), 57-64.

[15] Yusoff, M. S. B., Abdul Rahim, A. F., \& Yaacob, M. J. (2010). Prevalence and Sources of Stress among Universiti Sains Malaysia Medical Students. Malaysian Journal of Medical Sciences, 17(1), 3-11.

[16] Redhwan, A. A. N., Sami, A. R., Karim, A. J., Chan, R., \& Zaleha, M. I. (2009). Stress and coping strategies among management and science university students: A qualitative study. The International Medical Journal, 8(2), 11-16.

[17] McConnell, A. R., Brown, C. M., Shoda, T. M., Stayton, L. E., \& Martin, C. E. (2011). Friends with benefits: On the positive consequences of pet ownership. Journal of Personality and Social Psychology, 101(6), 1239-1252.

[18] Allen, K., Blascovich, J., \& Mendes, W. (2002). Cardiovascular reactivity and the presence of pets, friends, and spouses: The truth about cats and dogs. Psychosomatic Medicine, 64, 727-739.

[19] Brown, S. G., \& Rhodes, R. E. (2006). Relationships among dog ownership and leisure-time walking in Western Canadian adults. American Journal of Preventive Medicine, 30(2),131-136.

[20] Staats, S., Sears, K., \& Pierfelice, L. (2006). Teachers' pets and why they have them: An investigation of the human animal bond. Journal of Applied Social Psychology, 36(8), 1881-1891.

[21] Motooka, M., Koike, H., Yokoyama, T., \& Kennedy, N. L. (2006). Effect of dog-walking on autonomic nervous activity in senior citizens. Medical Journal of Australia, 184(2), 60-63.

[22] Siegel, J. M. (1990). Stressful life events and use of physician services among the elderly: The moderating role of pet ownership. Journal of Personality and Social Psychology, 58(6), 1081-1086.

[23] Barker, R. T., Knisely, J. S., Barker, S. B., Cobb, R. K., \& Schubert, C. M. (2012). Preliminary investigation of employee's dog presence on stress and organizational perceptions. International Journal of Workplace Health Management, 5(1), 15-30.

[24] Wells, M., \& Perrine, R. (2001). Critters in the cube farm: Perceived psychological and organizational effects of pets in the workplace. Journal of Occupational Health Psychology, 6(1), 81-87.

[25] Barker, S. B., Knisely, J. S., McCain, N. L., Schubert, C. M., \& Pandurangi, A. K. (2010). Exploratory study of stress-buffering response patterns from interaction with a therapy dog. Anthrozoös, 23(1), 79-91.
[26] Somervill, J. W., Kruglikova, Y. A., Robertson, R. L., Hanson, L. M., \& MacLin, O. H. (2008).Physiological responses by college students to a dog and a cat: Implications for pet therapy. North American Journal of Psychology, 10(3), 519-528.

[27] Lazarus, R. S. (1999). Stress and emotion: A new synthesis. New York: Springer.

[28] Matthieu, M. M., \& Ivanoff, A. (2006). Using stress, appraisal, and coping theories in clinical practice: Assessments of coping strategies after disasters. Brief Treatment and Crisis Intervention, 6(4), 337-348.

[29] Zimet, G. D., Dahlem, N. W., Zimet, S. G., \& Farley, G. K. (1988). The Multidimensional Scale of Perceived Social Support. Joumal of Personality Assessment, 52(1), 30-41.

[30] Andrews, J. P. (2011). Conversational elaboration and emotional well-being in perceived social support. McNair Scholars Journal, 15(1), 4-9.

[31] Ditzen, B., Schimidt, S., Strauss, B., Nater, U. M., Ehlert, U., \& Heinrichs, M. (2008). Adult attachment and social support interact to reduce psychological but not cortisol responses to stress. J Psychosom Res, 64(5), 479-86.

[32] Glozah, F. N. (2013). Effects of academic stress and perceived social support on the psychological wellbeing of adolescents in Ghana. Open Journal Medical Psychology, 2, 143-150.

[33] Hamdan-Mansour, A. M., \& Dawani, H. A. (2008). Social support and stress among university students in Jordan. Int $J$ Ment Health Addiction, 6, 442-450.

[34] Heinrichs, M., Baumgartner, T., Kirschbaum, C., \& Ehlert, U. (2003). Social support and oxytocin interact to suppress cortisol and subjective responses to psychosocial stress. Society of Biological Psychiatry, 54, 1389-1398.

[35] Chao, R. C. L. (2012). Managing perceived stress among college students: The roles of social support and dysfunctional coping. Journal of College Counseling, 15, 5-21.

[36] Lakey, B., \& Orehek, E. (2011). Relational regulation theory: A new approach to explain the link between perceived social support and mental health. Psychological Review, 118(3), $482-495$.

[37] Duvall Antonacopoulos, N. M., \& Pychyl, T. A. (2008). An examination of the relations between social support, anthropomorphism and stress among dog owners. ANTHROZOOS, 21(2), 139-152.

[38] Campo, R. A., \& Uchino, B. N. (2013). Humans' bonding with their campanion dogs: Cardiovascular benefits during and after stress. Journal of Sociology \& Social Welfare, XL(4), 237-259.

[39] Cohen, S. (1994). Perceived Stress Scale. Retrieved from http://wellness.pdx.edu/sites/default/files/PerceivedStressScal e.pdf

[40] Lai, C. S. (2014). College freshmen's self-efficacy, effort regulation, perceived stress and their adaptation to college. Asian Journal of Humanities and Social Sciences, 2(2), 107-117. 